\title{
Existence of nonoscillatory solutions tending to zero of fourth-order nonlinear neutral dynamic equations on time scales
}

\section{Yang-Cong Qiu ${ }^{*}$ (D)}

"Correspondence:

q840410@qq.com

'School of Humanities, Shunde

Polytechnic, Deshengdong Road,

528333 Foshan, P.R. China

\begin{abstract}
In this paper, a class of fourth-order nonlinear neutral dynamic equations on time scales is investigated. We obtain some sufficient conditions for the existence of nonoscillatory solutions tending to zero with some characteristics of the equations by Krasnoselskii's fixed point theorem. Finally, two interesting examples are presented to show the significance of the results.
\end{abstract}

MSC: 34N05; 34C10; 39A13

Keywords: Nonoscillatory solution; Tending to zero; Fourth-order; Neutral dynamic equation; Time scale

\section{Introduction}

In this paper, we consider the existence of nonoscillatory solutions tending to zero of a fourth-order nonlinear neutral dynamic equation

$$
R_{4}(t, x(t))+f(t, x(h(t)))=0
$$

on a time scale $\mathbb{T}$ with $\sup \mathbb{T}=\infty$, where

$$
R_{k}(t, x(t))= \begin{cases}x(t)+p(t) x(g(t)), & k=0, \\ r_{4-k}(t) R_{k-1}^{\Delta}(t, x(t)), & k=1,2,3, \\ R_{3}^{\Delta}(t, x(t)), & k=4\end{cases}
$$

and $t \in\left[t_{0}, \infty\right)_{\mathbb{T}}$ with $t_{0} \in \mathbb{T}$. Moreover, throughout this paper we satisfy the conditions as follows:

(C1) $r_{i} \in \mathrm{C}_{\mathrm{rd}}\left(\left[t_{0}, \infty\right)_{\mathbb{T}},(0, \infty)\right), i=1,2,3$;

(C2) $p \in \mathrm{C}_{\mathrm{rd}}\left(\left[t_{0}, \infty\right)_{\mathbb{T}},[0, \infty)\right)$ and $\lim _{t \rightarrow \infty} p(t)=p_{0} \in[0,1)$;

(C3) $g, h \in \mathrm{C}_{\mathrm{rd}}\left(\left[t_{0}, \infty\right)_{\mathbb{T}}, \mathbb{T}\right)$ and $\lim _{t \rightarrow \infty} g(t)=\lim _{t \rightarrow \infty} h(t)=\infty$;

(C4) $f \in \mathrm{C}\left(\left[t_{0}, \infty\right)_{\mathbb{T}} \times \mathbb{R}, \mathbb{R}\right)$ and $x f(t, x)>0$ for $x \neq 0$;

c) The Author(s) 2021. This article is licensed under a Creative Commons Attribution 4.0 International License, which permits use, sharing, adaptation, distribution and reproduction in any medium or format, as long as you give appropriate credit to the original author(s) and the source, provide a link to the Creative Commons licence, and indicate if changes were made. The images or other third party material in this article are included in the article's Creative Commons licence, unless indicated otherwise in a credit line to the material. If material is not included in the article's Creative Commons licence and your intended use is not permitted by statutory regulation or exceeds the permitted use, you will need to obtain permission directly from the copyright holder. To view a copy of this licence, visit http://creativecommons.org/licenses/by/4.0/. 
(C5) $\eta_{i}=\lim _{t \rightarrow \infty} H_{i}(g(t)) / H_{i}(t) \in(0,1]$, where

$$
H_{1}(t)=\int_{t}^{\infty} \frac{\Delta u_{3}}{r_{3}\left(u_{3}\right)}, \quad H_{2}(t)=\int_{t}^{\infty} \int_{u_{3}}^{\infty} \frac{\Delta u_{2} \Delta u_{3}}{r_{2}\left(u_{2}\right) r_{3}\left(u_{3}\right)},
$$

and

$$
H_{3}(t)=\int_{t}^{\infty} \int_{u_{3}}^{\infty} \int_{u_{2}}^{\infty} \frac{\Delta u_{1} \Delta u_{2} \Delta u_{3}}{r_{1}\left(u_{1}\right) r_{2}\left(u_{2}\right) r_{3}\left(u_{3}\right)}
$$

if $H_{i}\left(t_{0}\right)<\infty, i=1,2,3$, respectively.

In recent years, the research on nonoscillation of dynamic equations on time scales has made some progress. The scientists have provided some sufficient conditions which guarantee that the equations have nonoscillatory solutions with certain characteristics. We refer the reader to [1-6] for details of the theory of time scale, and [7-22] with the references cited therein for the achievements on the existence of nonoscillatory solutions of nonlinear neutral dynamic equations on time scales.

A solution $x$ of (1) is called eventually positive (or eventually negative) if there exists $T \in\left[t_{0}, \infty\right)_{\mathbb{T}}$ satisfying $x(t)>0($ or $x(t)<0)$ for $t \in[T, \infty)_{\mathbb{T}}$. The existence and asymptotic behavior of nonoscillatory solutions of a class of nonlinear neutral dynamic equations on time scales similar to (1) have been studied successively. Without loss of generality, only the eventually positive solutions are considered. For the first-order case, Zhu and Wang [22] investigated

$$
(x(t)+p(t) x(g(t)))^{\Delta}+f(t, x(h(t)))=0 .
$$

Gao and Wang [8], Deng and Wang [7] considered the second-order case

$$
\left(r(t)(x(t)+p(t) x(g(t)))^{\Delta}\right)^{\Delta}+f(t, x(h(t)))=0
$$

under different assumptions $\int_{t_{0}}^{\infty} 1 / r(t) \Delta t<\infty$ and $\int_{t_{0}}^{\infty} 1 / r(t) \Delta t=\infty$, respectively. Then, the third-order case

$$
\left(r_{1}(t)\left(r_{2}(t)(x(t)+p(t) x(g(t)))^{\Delta}\right)^{\Delta}\right)^{\Delta}+f(t, x(h(t)))=0
$$

was studied in $[15,19,21]$, and the higher-order case was considered in $[17,18,20]$. To have a deeper understanding of the asymptotic behavior of nonoscillatory solutions of these equations, Qiu [16] studied (1) with some conditions. In their works, different groups of eventually positive solutions of the equations are summarized. For each case, an appropriate Banach space is introduced and Krasnoselskii's fixed point theorem is employed to present some sufficient conditions (or necessary and sufficient conditions) for the existence of these solutions.

We note that the case tending to zero is an important type for nonoscillatory solutions of the equations. However, the asymptotic behavior of this type is more complicated than those of other cases. It is obvious that the results of the existence for nonoscillatory solutions tending to zero are not satisfactory in $[7,8,15,20,22]$. Some special sufficient conditions are provided but not enough to be applied universally. Therefore, new methods 
should be found to study nonoscillatory solutions tending to zero of the equations. Mojsej and Tartal'ová [23] were concerned with a third-order nonlinear differential equation

$$
\left(\frac{1}{p(t)}\left(\frac{1}{r(t)} x^{\prime}(t)\right)^{\prime}\right)^{\prime}+q(t) f(x(t))=0, \quad t \geq a,
$$

where $f$ satisfies the Lipschitz condition. The authors obtained some nice sufficient conditions to ensure that (3) has a solution $x$ with $\lim _{t \rightarrow \infty} x(t)=0$ meeting some characteristics. Inspired by [23], Qiu [24] investigated the nonoscillatory solutions tending to zero of (2) when $g(t) \geq t$ for $t \in\left[t_{0}, \infty\right)_{\mathbb{T}}$, by employing a Banach space

$$
\mathrm{BC}\left[T_{0}, \infty\right)_{\mathbb{T}}=\left\{x \in \mathrm{C}\left(\left[T_{0}, \infty\right)_{\mathbb{T}}, \mathbb{R}\right): \sup _{t \in\left[T_{0}, \infty\right)_{\mathbb{T}}}|x(t)|<\infty\right\},
$$

where $C\left(\left[T_{0}, \infty\right)_{\mathbb{T}}, \mathbb{R}\right)$ is the set of all continuous functions that map $\left[T_{0}, \infty\right)_{\mathbb{T}}$ into $\mathbb{R}$ and $\|x\|=\sup _{t \in\left[T_{0}, \infty\right)_{\mathbb{T}}}|x(t)|$. According to Krasnoselskii's fixed point theorem, some new results are presented. However, considering the cases such as $g(t)=t-2, g(t)=t / 3$, and $g(t)=t+\cos t$ for $t \in\left[t_{0}, \infty\right)_{\mathbb{T}}$, the conclusions in [24] are not applicable when $g(t) \geq t$ is not fulfilled eventually, especially for [7, 8, 15-22]. Afterwards, Qiu et al. [25] studied (2) under $g(t) \leq t$ for $t \in\left[t_{0}, \infty\right)_{\mathbb{T}}$ and partially solved the problem. In this paper, we continue to relax the constraint and unite the cases of the function $g$. Provided that $H_{i}$ have been defined for $i=1,2,3$, note that they are all strictly decreasing on $\left[t_{0}, \infty\right)_{\mathbb{T}}$. For the case that $g(t) \geq t$ is not satisfied eventually, the condition $\eta_{i}=1$ should be satisfied for $i=1,2,3$, respectively.

In the following, Krasnoselskii's fixed point theorem (see [26]) is presented in Lemma 1.1, which will be used in the next section. Then, we show the relation between $R_{0}$ and $x$ in Lemma 1.2 (see [24, Lemma 2.5]).

Lemma 1.1 Suppose that $U$ is a contraction mapping, $V$ is completely continuous, and $U x+V y \in \Omega$ holds for all $x, y \in \Omega$, where $U, V: \Omega \rightarrow X$ are two operators, $X$ is a Banach space, and $\Omega$ is a bounded, convex, and closed subset of $X$, then $U+V$ has a fixed point in $\Omega$.

Lemma 1.2 Suppose that $x$ is an eventually positive solution of (1). If there exists a constant $a \geq 0$ satisfying $\lim _{t \rightarrow \infty} R_{0}(t, x(t))=a$, then we have

$$
\lim _{t \rightarrow \infty} x(t)=\frac{a}{1+p_{0}} .
$$

\section{Main results}

In this section, we present some sufficient conditions for the existence of eventually positive solutions of (1) under different assumptions. Firstly, suppose that the function $f(t, x)$ is nondecreasing with respect to $x$, then we have Theorems 2.1-2.4.

Theorem 2.1 Assume that the function $f(t, x)$ is nondecreasing with respect to $x, H_{1}\left(t_{0}\right)<$ $\infty$, and

$$
\int_{t_{0}}^{\infty} \int_{t_{0}}^{u_{2}} \int_{t_{0}}^{u_{1}} \frac{f\left(u_{0}, 2 H_{1}\left(h\left(u_{0}\right)\right)\right)}{r_{1}\left(u_{1}\right) r_{2}\left(u_{2}\right)} \Delta u_{0} \Delta u_{1} \Delta u_{2}<\infty,
$$


then there exists $T_{1} \in\left[t_{0}, \infty\right)_{\mathbb{T}}$ such that (1) has two eventually positive solutions $x_{1}$ and $x_{2}$ tending to zero, which satisfy that $R_{0}\left(t, x_{i}(t)\right)>0, R_{1}\left(t, x_{i}(t)\right)<0, i=1,2, R_{2}\left(t, x_{1}(t)\right)<0$, $R_{3}\left(t, x_{1}(t)\right)<0, R_{2}\left(t, x_{2}(t)\right)>0$, and $R_{3}\left(t, x_{2}(t)\right)>0$ for $t \in\left[T_{1}, \infty\right)_{\mathbb{T}}$.

Proof Take $p_{1}$ satisfying $p_{0}<p_{1}<\left(1+4 p_{0}\right) / 5<1$, then there exists $T_{0} \in\left[t_{0}, \infty\right)_{\mathbb{T}}$ such that

$$
\frac{5 p_{1}-1}{4} \leq p(t) \leq p_{1}<1, \quad p(t) \frac{H_{1}(g(t))}{H_{1}(t)} \geq \frac{\left(5 p_{1}-1\right) \eta_{1}}{4}, \quad t \in\left[T_{0}, \infty\right)_{\mathbb{T}}
$$

and

$$
\int_{T_{0}}^{\infty} \int_{T_{0}}^{u_{2}} \int_{T_{0}}^{u_{1}} \frac{f\left(u_{0}, 2 H_{1}\left(h\left(u_{0}\right)\right)\right)}{r_{1}\left(u_{1}\right) r_{2}\left(u_{2}\right)} \Delta u_{0} \Delta u_{1} \Delta u_{2} \leq \frac{1-p_{1} \eta_{1}}{4} .
$$

Choose $T_{1} \in\left(T_{0}, \infty\right)_{\mathbb{T}}$ such that $g(t) \geq T_{0}$ and $h(t) \geq T_{0}$ for $t \in\left[T_{1}, \infty\right)_{\mathbb{T}}$. Define a Banach space $\mathrm{BC}\left[T_{0}, \infty\right)_{\mathbb{T}}$ as $(4), \Omega_{1}=\left\{x \in \mathrm{BC}\left[T_{0}, \infty\right)_{\mathbb{T}}: H_{1}(t) \leq x(t) \leq 2 H_{1}(t)\right\}$, and two operators $U_{1}, V_{1}: \Omega_{1} \rightarrow \mathrm{BC}\left[T_{0}, \infty\right)_{\mathbb{T}}$ as follows:

$$
\begin{aligned}
& \left(U_{1} x\right)(t)= \begin{cases}\left(U_{1} x\right)\left(T_{1}\right), & t \in\left[T_{0}, T_{1}\right)_{\mathbb{T}}, \\
3 p_{1} \eta_{1} H_{1}(t) / 2-p(t) x(g(t)), & t \in\left[T_{1}, \infty\right)_{\mathbb{T}},\end{cases} \\
& \left(V_{1} x\right)(t)= \begin{cases}\left(V_{1} x\right)\left(T_{1}\right), & t \in\left[T_{0}, T_{1}\right)_{\mathbb{T}}, \\
3 H_{1}(t) / 2 & \\
+\int_{t}^{\infty} \int_{T_{1}}^{u_{3}} \int_{T_{1}}^{u_{2}} \int_{T_{1}}^{u_{1}} \frac{f\left(u_{0}, x\left(h\left(u_{0}\right)\right)\right)}{r_{1}\left(u_{1}\right) r_{2}\left(u_{2}\right) r_{3}\left(u_{3}\right)} \Delta u_{0} \Delta u_{1} \Delta u_{2} \Delta u_{3}, & t \in\left[T_{1}, \infty\right)_{\mathbb{T}} .\end{cases}
\end{aligned}
$$

The proof that $U_{1}$ and $V_{1}$ satisfy the conditions in Lemma 1.1 is similar to those of [7, Theorem 2.5], [8, Theorem 2], [15, Theorem 3.1], and [22, Theorem 8], so it is omitted here. Therefore, there exists $x_{1} \in \Omega_{1}$ such that $\left(U_{1}+V_{1}\right) x_{1}=x_{1}$, and then, for $t \in\left[T_{1}, \infty\right)_{\mathbb{T}}$, we obtain

$$
\begin{aligned}
x_{1}(t)= & \frac{3\left(1+p_{1} \eta_{1}\right)}{2} H_{1}(t)-p(t) x_{1}(g(t)) \\
& +\int_{t}^{\infty} \int_{T_{1}}^{u_{3}} \int_{T_{1}}^{u_{2}} \int_{T_{1}}^{u_{1}} \frac{f\left(u_{0}, x_{1}\left(h\left(u_{0}\right)\right)\right)}{r_{1}\left(u_{1}\right) r_{2}\left(u_{2}\right) r_{3}\left(u_{3}\right)} \Delta u_{0} \Delta u_{1} \Delta u_{2} \Delta u_{3} .
\end{aligned}
$$

Since

$$
\begin{gathered}
\int_{t}^{\infty} \int_{T_{1}}^{u_{3}} \int_{T_{1}}^{u_{2}} \int_{T_{1}}^{u_{1}} \frac{f\left(u_{0}, x_{1}\left(h\left(u_{0}\right)\right)\right)}{r_{1}\left(u_{1}\right) r_{2}\left(u_{2}\right) r_{3}\left(u_{3}\right)} \Delta u_{0} \Delta u_{1} \Delta u_{2} \Delta u_{3} \\
<H_{1}(t) \int_{T_{1}}^{\infty} \int_{T_{1}}^{u_{2}} \int_{T_{1}}^{u_{1}} \frac{f\left(u_{0}, x_{1}\left(h\left(u_{0}\right)\right)\right)}{r_{1}\left(u_{1}\right) r_{2}\left(u_{2}\right)} \Delta u_{0} \Delta u_{1} \Delta u_{2} \\
\leq H_{1}(t) \int_{T_{1}}^{\infty} \int_{T_{1}}^{u_{2}} \int_{T_{1}}^{u_{1}} \frac{f\left(u_{0}, 2 H_{1}\left(h\left(u_{0}\right)\right)\right)}{r_{1}\left(u_{1}\right) r_{2}\left(u_{2}\right)} \Delta u_{0} \Delta u_{1} \Delta u_{2}
\end{gathered}
$$

for $t \in\left[T_{1}, \infty\right)_{\mathbb{T}}$ and

$$
\lim _{t \rightarrow \infty} H_{1}(t) \int_{T_{1}}^{\infty} \int_{T_{1}}^{u_{2}} \int_{T_{1}}^{u_{1}} \frac{f\left(u_{0}, 2 H_{1}\left(h\left(u_{0}\right)\right)\right)}{r_{1}\left(u_{1}\right) r_{2}\left(u_{2}\right)} \Delta u_{0} \Delta u_{1} \Delta u_{2}=0
$$


in view of (5), by Lemma 1.2 , we derive

$$
\lim _{t \rightarrow \infty} x_{1}(t)=\lim _{t \rightarrow \infty} R_{0}\left(t, x_{1}(t)\right)=0
$$

Moreover, for $t \in\left[T_{1}, \infty\right)_{\mathbb{T}}$, it follows that

$$
\begin{aligned}
R_{0}\left(t, x_{1}(t)\right)= & \frac{3\left(1+p_{1} \eta_{1}\right)}{2} H_{1}(t) \\
& +\int_{t}^{\infty} \int_{T_{1}}^{u_{3}} \int_{T_{1}}^{u_{2}} \int_{T_{1}}^{u_{1}} \frac{f\left(u_{0}, x_{1}\left(h\left(u_{0}\right)\right)\right)}{r_{1}\left(u_{1}\right) r_{2}\left(u_{2}\right) r_{3}\left(u_{3}\right)} \Delta u_{0} \Delta u_{1} \Delta u_{2} \Delta u_{3}>0 \\
R_{1}\left(t, x_{1}(t)\right)= & -\frac{3\left(1+p_{1} \eta_{1}\right)}{2} \\
& -\int_{T_{1}}^{t} \int_{T_{1}}^{u_{2}} \int_{T_{1}}^{u_{1}} \frac{f\left(u_{0}, x_{1}\left(h\left(u_{0}\right)\right)\right)}{r_{1}\left(u_{1}\right) r_{2}\left(u_{2}\right)} \Delta u_{0} \Delta u_{1} \Delta u_{2}<0, \\
R_{2}\left(t, x_{1}(t)\right)= & -\int_{T_{1}}^{t} \int_{T_{1}}^{u_{1}} \frac{f\left(u_{0}, x_{1}\left(h\left(u_{0}\right)\right)\right)}{r_{1}\left(u_{1}\right)} \Delta u_{0} \Delta u_{1}<0,
\end{aligned}
$$

and

$$
R_{3}\left(t, x_{1}(t)\right)=-\int_{T_{1}}^{t} f\left(u_{0}, x\left(h\left(u_{0}\right)\right)\right) \Delta u_{0}<0 .
$$

On the other hand, we define another operator $\bar{V}_{1}: \Omega_{1} \rightarrow \mathrm{BC}\left[T_{0}, \infty\right)_{\mathbb{T}}$ as follows:

$$
\left(\bar{V}_{1} x\right)(t)= \begin{cases}\left(\bar{V}_{1} x\right)\left(T_{1}\right), & t \in\left[T_{0}, T_{1}\right)_{\mathbb{T}}, \\ 3 H_{1}(t) / 2 & \\ \quad+\int_{t}^{\infty} \int_{u_{3}}^{\infty} \int_{T_{1}}^{u_{2}} \int_{T_{1}}^{u_{1}} \frac{f\left(u_{0}, x\left(h\left(u_{0}\right)\right)\right)}{r_{1}\left(u_{1}\right) r_{2}\left(u_{2}\right) r_{3}\left(u_{3}\right)} \Delta u_{0} \Delta u_{1} \Delta u_{2} \Delta u_{3}, & t \in\left[T_{1}, \infty\right)_{\mathbb{T}} .\end{cases}
$$

Similarly, there exists $x_{2} \in \Omega_{1}$ such that $\left(U_{1}+\bar{V}_{1}\right) x_{2}=x_{2}$, and then, for $t \in\left[T_{1}, \infty\right)_{\mathbb{T}}$, we obtain

$$
\begin{aligned}
x_{2}(t)= & \frac{3\left(1+p_{1} \eta_{1}\right)}{2} H_{1}(t)-p(t) x_{2}(g(t)) \\
& +\int_{t}^{\infty} \int_{u_{3}}^{\infty} \int_{T_{1}}^{u_{2}} \int_{T_{1}}^{u_{1}} \frac{f\left(u_{0}, x_{2}\left(h\left(u_{0}\right)\right)\right)}{r_{1}\left(u_{1}\right) r_{2}\left(u_{2}\right) r_{3}\left(u_{3}\right)} \Delta u_{0} \Delta u_{1} \Delta u_{2} \Delta u_{3} .
\end{aligned}
$$

It follows that

$$
\lim _{t \rightarrow \infty} x_{2}(t)=\lim _{t \rightarrow \infty} R_{0}\left(t, x_{2}(t)\right)=0
$$

For $t \in\left[T_{1}, \infty\right)_{\mathbb{T}}$, we obtain

$$
R_{0}\left(t, x_{2}(t)\right)>0, \quad R_{1}\left(t, x_{2}(t)\right)<0, \quad R_{2}\left(t, x_{2}(t)\right)>0, \quad R_{3}\left(t, x_{2}(t)\right)>0 .
$$

The proof is complete. 
Theorem 2.2 Assume that the function $f(t, x)$ is nondecreasing with respect to $x, H_{1}\left(t_{0}\right)<$ $\infty$, and

$$
\int_{t_{0}}^{\infty} \int_{u_{2}}^{\infty} \int_{t_{0}}^{u_{1}} \frac{f\left(u_{0}, 2 H_{1}\left(h\left(u_{0}\right)\right)\right)}{r_{1}\left(u_{1}\right) r_{2}\left(u_{2}\right)} \Delta u_{0} \Delta u_{1} \Delta u_{2}<\infty,
$$

then there exists $T_{1} \in\left[t_{0}, \infty\right)_{\mathbb{T}}$ such that (1) has two eventually positive solutions $x_{1}$ and $x_{2}$ tending to zero, which satisfy that $R_{0}\left(t, x_{i}(t)\right)>0, R_{1}\left(t, x_{i}(t)\right)<0, i=1,2, R_{2}\left(t, x_{1}(t)\right)<0$, $R_{3}\left(t, x_{1}(t)\right)>0, R_{2}\left(t, x_{2}(t)\right)>0$, and $R_{3}\left(t, x_{2}(t)\right)<0$ for $t \in\left[T_{1}, \infty\right)_{\mathbb{T}}$.

Proof Take $p_{1}$ satisfying $p_{0}<p_{1}<\left(1+4 p_{0}\right) / 5<1$, then there exists $T_{0} \in\left[t_{0}, \infty\right)_{\mathbb{T}}$ such that (6) holds and

$$
\int_{T_{0}}^{\infty} \int_{u_{2}}^{\infty} \int_{T_{0}}^{u_{1}} \frac{f\left(u_{0}, 2 H_{1}\left(h\left(u_{0}\right)\right)\right)}{r_{1}\left(u_{1}\right) r_{2}\left(u_{2}\right)} \Delta u_{0} \Delta u_{1} \Delta u_{2} \leq \frac{1-p_{1} \eta_{1}}{4}
$$

Define the same $T_{1}, \mathrm{BC}\left[T_{0}, \infty\right)_{\mathbb{T}}, \Omega_{1}$, and $U_{1}$ as in Theorem 2.1, and an operator $V_{1}^{\prime}: \Omega_{1} \rightarrow$ $\mathrm{BC}\left[T_{0}, \infty\right)_{\mathbb{T}}$ as follows:

$$
\left(V_{1}^{\prime} x\right)(t)= \begin{cases}\left(V_{1}^{\prime} x\right)\left(T_{1}\right), & t \in\left[T_{0}, T_{1}\right)_{\mathbb{T}}, \\ 3 H_{1}(t) / 2 & \\ \quad+\int_{t}^{\infty} \int_{T_{1}}^{u_{3}} \int_{u_{2}}^{\infty} \int_{T_{1}}^{u_{1}} \frac{f\left(u_{0}, x\left(h\left(u_{0}\right)\right)\right)}{r_{1}\left(u_{1}\right) r_{2}\left(u_{2}\right) r_{3}\left(u_{3}\right)} \Delta u_{0} \Delta u_{1} \Delta u_{2} \Delta u_{3}, & t \in\left[T_{1}, \infty\right)_{\mathbb{T}} .\end{cases}
$$

Then there exists $x_{1} \in \Omega_{1}$ such that $\left(U_{1}+V_{1}^{\prime}\right) x_{1}=x_{1}$. For $t \in\left[T_{1}, \infty\right)_{\mathbb{T}}$, we obtain

$$
\begin{aligned}
x_{1}(t)= & \frac{3\left(1+p_{1} \eta_{1}\right)}{2} H_{1}(t)-p(t) x_{1}(g(t)) \\
& +\int_{t}^{\infty} \int_{T_{1}}^{u_{3}} \int_{u_{2}}^{\infty} \int_{T_{1}}^{u_{1}} \frac{f\left(u_{0}, x_{1}\left(h\left(u_{0}\right)\right)\right)}{r_{1}\left(u_{1}\right) r_{2}\left(u_{2}\right) r_{3}\left(u_{3}\right)} \Delta u_{0} \Delta u_{1} \Delta u_{2} \Delta u_{3},
\end{aligned}
$$

which means that (8) and

$$
R_{0}\left(t, x_{1}(t)\right)>0, \quad R_{1}\left(t, x_{1}(t)\right)<0, \quad R_{2}\left(t, x_{1}(t)\right)<0, \quad R_{3}\left(t, x_{1}(t)\right)>0
$$

for $t \in\left[T_{1}, \infty\right)_{\mathbb{T}}$.

Define another operator $\bar{V}_{1}^{\prime}: \Omega_{1} \rightarrow \mathrm{BC}\left[T_{0}, \infty\right)_{\mathbb{T}}$ as follows:

$$
\left(\bar{V}_{1}^{\prime} x\right)(t)= \begin{cases}\left(\bar{V}_{1}^{\prime} x\right)\left(T_{1}\right), & t \in\left[T_{0}, T_{1}\right)_{\mathbb{T}}, \\ 3 H_{1}(t) / 2 & \\ \quad+\int_{t}^{\infty} \int_{u_{3}}^{\infty} \int_{u_{2}}^{\infty} \int_{T_{1}}^{u_{1}} \frac{f\left(u_{0}, x\left(h\left(u_{0}\right)\right)\right)}{r_{1}\left(u_{1}\right) r_{2}\left(u_{2}\right) r_{3}\left(u_{3}\right)} \Delta u_{0} \Delta u_{1} \Delta u_{2} \Delta u_{3}, & t \in\left[T_{1}, \infty\right)_{\mathbb{T}} .\end{cases}
$$

Then there exists $x_{2} \in \Omega_{1}$ such that $\left(U_{1}+\bar{V}_{1}^{\prime}\right) x_{2}=x_{2}$, and then, for $t \in\left[T_{1}, \infty\right)_{\mathbb{T}}$, we obtain

$$
\begin{aligned}
x_{2}(t)= & \frac{3\left(1+p_{1} \eta_{1}\right)}{2} H_{1}(t)-p(t) x_{2}(g(t)) \\
& +\int_{t}^{\infty} \int_{u_{3}}^{\infty} \int_{u_{2}}^{\infty} \int_{T_{1}}^{u_{1}} \frac{f\left(u_{0}, x_{2}\left(h\left(u_{0}\right)\right)\right)}{r_{1}\left(u_{1}\right) r_{2}\left(u_{2}\right) r_{3}\left(u_{3}\right)} \Delta u_{0} \Delta u_{1} \Delta u_{2} \Delta u_{3} .
\end{aligned}
$$


It follows that (9) holds and

$$
R_{0}\left(t, x_{2}(t)\right)>0, \quad R_{1}\left(t, x_{2}(t)\right)<0, \quad R_{2}\left(t, x_{2}(t)\right)>0, \quad R_{3}\left(t, x_{2}(t)\right)>0
$$

for $t \in\left[T_{1}, \infty\right)_{\mathbb{T}}$. This completes the proof.

Theorem 2.3 Assume that the function $f(t, x)$ is nondecreasing with respect to $x, H_{2}\left(t_{0}\right)<$ $\infty$, and

$$
\int_{t_{0}}^{\infty} \int_{t_{0}}^{u_{1}} \frac{f\left(u_{0}, 2 H_{2}\left(h\left(u_{0}\right)\right)\right)}{r_{1}\left(u_{1}\right)} \Delta u_{0} \Delta u_{1}<\infty
$$

then there exists $T_{1} \in\left[t_{0}, \infty\right)_{\mathbb{T}}$ such that (1) has two eventually positive solutions $x_{1}$ and $x_{2}$ tending to zero, which satisfy that $R_{0}\left(t, x_{i}(t)\right)>0, R_{1}\left(t, x_{i}(t)\right)<0, R_{2}\left(t, x_{i}(t)\right)>0, i=1,2$, $R_{3}\left(t, x_{1}(t)\right)>0$, and $R_{3}\left(t, x_{2}(t)\right)<0$ for $t \in\left[T_{1}, \infty\right)_{\mathbb{T}}$.

Proof Take $p_{1}$ as in Theorem 2.1. Then there exists $T_{0} \in\left[t_{0}, \infty\right)_{\mathbb{T}}$ such that

$$
\frac{5 p_{1}-1}{4} \leq p(t) \leq p_{1}<1, \quad p(t) \frac{H_{2}(g(t))}{H_{2}(t)} \geq \frac{\left(5 p_{1}-1\right) \eta_{2}}{4}, \quad t \in\left[T_{0}, \infty\right)_{\mathbb{T}}
$$

and

$$
\int_{T_{0}}^{\infty} \int_{T_{0}}^{u_{1}} \frac{f\left(u_{0}, 2 H_{2}\left(h\left(u_{0}\right)\right)\right)}{r_{1}\left(u_{1}\right)} \Delta u_{0} \Delta u_{1} \leq \frac{1-p_{1} \eta_{2}}{4} .
$$

Choose the same $T_{1}, \mathrm{BC}\left[T_{0}, \infty\right)_{\mathbb{T}}$ as in Theorem 2.1, $\Omega_{2}=\left\{x \in \mathrm{BC}\left[T_{0}, \infty\right)_{\mathbb{T}}: H_{2}(t) \leq x(t) \leq\right.$ $\left.2 H_{2}(t)\right\}$, and two operators $U_{2}, V_{2}: \Omega_{2} \rightarrow \mathrm{BC}\left[T_{0}, \infty\right)_{\mathbb{T}}$ as follows:

$$
\begin{aligned}
& \left(U_{2} x\right)(t)= \begin{cases}\left(U_{2} x\right)\left(T_{1}\right), & t \in\left[T_{0}, T_{1}\right)_{\mathbb{T}}, \\
3 p_{1} \eta_{2} H_{2}(t) / 2-p(t) x(g(t)), & t \in\left[T_{1}, \infty\right)_{\mathbb{T}},\end{cases} \\
& \left(V_{2} x\right)(t)= \begin{cases}\left(V_{2} x\right)\left(T_{1}\right), & t \in\left[T_{0}, T_{1}\right)_{\mathbb{T}}, \\
3 H_{2}(t) / 2 & \\
\quad+\int_{t}^{\infty} \int_{u_{3}}^{\infty} \int_{T_{1}}^{u_{2}} \int_{T_{1}}^{u_{1}} \frac{f\left(u_{0}, x\left(h\left(u_{0}\right)\right)\right)}{r_{1}\left(u_{1}\right) r_{2}\left(u_{2}\right) r_{3}\left(u_{3}\right)} \Delta u_{0} \Delta u_{1} \Delta u_{2} \Delta u_{3}, & t \in\left[T_{1}, \infty\right)_{\mathbb{T}} .\end{cases}
\end{aligned}
$$

Similarly, $U_{2}$ and $V_{2}$ satisfy the conditions in Lemma 1.1. Then there exists $x_{1} \in \Omega_{2}$ such that $\left(U_{2}+V_{2}\right) x_{1}=x_{1}$. For $t \in\left[T_{1}, \infty\right)_{\mathbb{T}}$, it follows that

$$
\begin{aligned}
x_{1}(t)= & \frac{3\left(1+p_{1} \eta_{2}\right)}{2} H_{2}(t)-p(t) x_{1}(g(t)) \\
& +\int_{t}^{\infty} \int_{u_{3}}^{\infty} \int_{T_{1}}^{u_{2}} \int_{T_{1}}^{u_{1}} \frac{f\left(u_{0}, x_{1}\left(h\left(u_{0}\right)\right)\right)}{r_{1}\left(u_{1}\right) r_{2}\left(u_{2}\right) r_{3}\left(u_{3}\right)} \Delta u_{0} \Delta u_{1} \Delta u_{2} \Delta u_{3} .
\end{aligned}
$$

Since

$$
\begin{gathered}
\int_{t}^{\infty} \int_{u_{3}}^{\infty} \int_{T_{1}}^{u_{2}} \int_{T_{1}}^{u_{1}} \frac{f\left(u_{0}, x_{1}\left(h\left(u_{0}\right)\right)\right)}{r_{1}\left(u_{1}\right) r_{2}\left(u_{2}\right) r_{3}\left(u_{3}\right)} \Delta u_{0} \Delta u_{1} \Delta u_{2} \Delta u_{3} \\
<H_{2}(t) \int_{T_{1}}^{\infty} \int_{T_{1}}^{u_{1}} \frac{f\left(u_{0}, x_{1}\left(h\left(u_{0}\right)\right)\right)}{r_{1}\left(u_{1}\right) r_{2}\left(u_{2}\right)} \Delta u_{0} \Delta u_{1}
\end{gathered}
$$




$$
\leq H_{2}(t) \int_{T_{1}}^{\infty} \int_{T_{1}}^{u_{1}} \frac{f\left(u_{0}, 2 H_{2}\left(h\left(u_{0}\right)\right)\right)}{r_{1}\left(u_{1}\right) r_{2}\left(u_{2}\right)} \Delta u_{0} \Delta u_{1}
$$

for $t \in\left[T_{1}, \infty\right)_{\mathbb{T}}$ and

$$
\lim _{t \rightarrow \infty} H_{2}(t) \int_{T_{1}}^{\infty} \int_{T_{1}}^{u_{1}} \frac{f\left(u_{0}, 2 H_{2}\left(h\left(u_{0}\right)\right)\right)}{r_{1}\left(u_{1}\right) r_{2}\left(u_{2}\right)} \Delta u_{0} \Delta u_{1}=0
$$

by virtue of (11), by Lemma 1.2, we obtain (8) and

$$
R_{0}\left(t, x_{1}(t)\right)>0, \quad R_{1}\left(t, x_{1}(t)\right)<0, \quad R_{2}\left(t, x_{1}(t)\right)>0, \quad R_{3}\left(t, x_{1}(t)\right)>0
$$

for $t \in\left[T_{1}, \infty\right)_{\mathbb{T}}$.

On the other hand, define $\bar{V}_{2}: \Omega_{2} \rightarrow \mathrm{BC}\left[T_{0}, \infty\right)_{\mathbb{T}}$ as follows:

$$
\left(\bar{V}_{2} x\right)(t)= \begin{cases}\left(\bar{V}_{2} x\right)\left(T_{1}\right), & t \in\left[T_{0}, T_{1}\right)_{\mathbb{T}}, \\ 3 H_{2}(t) / 2 & \\ \quad+\int_{t}^{\infty} \int_{u_{3}}^{\infty} \int_{u_{2}}^{\infty} \int_{T_{1}}^{u_{1}} \frac{f\left(u_{0}, x\left(h\left(u_{0}\right)\right)\right)}{r_{1}\left(u_{1}\right) r_{2}\left(u_{2}\right) r_{3}\left(u_{3}\right)} \Delta u_{0} \Delta u_{1} \Delta u_{2} \Delta u_{3}, & t \in\left[T_{1}, \infty\right)_{\mathbb{T}} .\end{cases}
$$

Similarly, there exists $x_{2} \in \Omega_{2}$ such that $\left(U_{2}+\bar{V}_{2}\right) x_{2}=x_{2}$, and then, for $t \in\left[T_{1}, \infty\right)_{\mathbb{T}}$, we obtain

$$
\begin{aligned}
x_{2}(t)= & \frac{3\left(1+p_{1} \eta_{2}\right)}{2} H_{2}(t)-p(t) x_{2}(g(t)) \\
& +\int_{t}^{\infty} \int_{u_{3}}^{\infty} \int_{u_{2}}^{\infty} \int_{T_{1}}^{u_{1}} \frac{f\left(u_{0}, x_{2}\left(h\left(u_{0}\right)\right)\right)}{r_{1}\left(u_{1}\right) r_{2}\left(u_{2}\right) r_{3}\left(u_{3}\right)} \Delta u_{0} \Delta u_{1} \Delta u_{2} \Delta u_{3},
\end{aligned}
$$

which implies that (9) holds. For $t \in\left[T_{1}, \infty\right)_{\mathbb{T}}$, we derive

$$
R_{0}\left(t, x_{2}(t)\right)>0, \quad R_{1}\left(t, x_{2}(t)\right)<0, \quad R_{2}\left(t, x_{2}(t)\right)>0, \quad R_{3}\left(t, x_{2}(t)\right)<0 .
$$

The proof is complete.

Theorem 2.4 Assume that the function $f(t, x)$ is nondecreasing with respect to $x, H_{3}\left(t_{0}\right)<$ $\infty$, and

$$
\int_{t_{0}}^{\infty} f\left(u_{0}, 2 H_{3}\left(h\left(u_{0}\right)\right)\right) \Delta u_{0}<\infty
$$

then there exists $T_{1} \in\left[t_{0}, \infty\right)_{\mathbb{T}}$ such that (1) has an eventually positive solution $x$ tending to zero, which satisfies that $R_{0}(t, x(t))>0, R_{1}(t, x(t))<0, R_{2}(t, x(t))>0$, and $R_{3}(t, x(t))<0$ for $t \in\left[T_{1}, \infty\right)_{\mathbb{T}}$.

Proof Take $p_{1}$ as in Theorem 2.1. Then there exists $T_{0} \in\left[t_{0}, \infty\right)_{\mathbb{T}}$ such that

$$
\frac{5 p_{1}-1}{4} \leq p(t) \leq p_{1}<1, \quad p(t) \frac{H_{3}(g(t))}{H_{3}(t)} \geq \frac{\left(5 p_{1}-1\right) \eta_{3}}{4}, \quad t \in\left[T_{0}, \infty\right)_{\mathbb{T}}
$$

and

$$
\int_{T_{0}}^{\infty} f\left(u_{0}, 2 H_{3}\left(h\left(u_{0}\right)\right)\right) \Delta u_{0} \leq \frac{1-p_{1} \eta_{3}}{4} .
$$


Choose the same $T_{1}, \mathrm{BC}\left[T_{0}, \infty\right)_{\mathbb{T}}$ as in Theorem 2.1, $\Omega_{3}=\left\{x \in \mathrm{BC}\left[T_{0}, \infty\right)_{\mathbb{T}}: H_{3}(t) \leq x(t) \leq\right.$ $\left.2 H_{3}(t)\right\}$, and two operators $U_{3}, V_{3}: \Omega_{3} \rightarrow \mathrm{BC}\left[T_{0}, \infty\right)_{\mathbb{T}}$ as follows:

$$
\begin{aligned}
& \left(U_{3} x\right)(t)= \begin{cases}\left(U_{3} x\right)\left(T_{1}\right), & t \in\left[T_{0}, T_{1}\right)_{\mathbb{T}}, \\
3 p_{1} \eta_{3} H_{3}(t) / 2-p(t) x(g(t)), & t \in\left[T_{1}, \infty\right)_{\mathbb{T}},\end{cases} \\
& \left(V_{3} x\right)(t)= \begin{cases}\left(V_{3} x\right)\left(T_{1}\right), & t \in\left[T_{0}, T_{1}\right)_{\mathbb{T}}, \\
3 H_{3}(t) / 2 & \\
\quad+\int_{t}^{\infty} \int_{u_{3}}^{\infty} \int_{u_{2}}^{\infty} \int_{T_{1}}^{u_{1}} \frac{f\left(u_{0}, x\left(h\left(u_{0}\right)\right)\right)}{r_{1}\left(u_{1}\right) r_{2}\left(u_{2}\right) r_{3}\left(u_{3}\right)} \Delta u_{0} \Delta u_{1} \Delta u_{2} \Delta u_{3}, & t \in\left[T_{1}, \infty\right)_{\mathbb{T}} .\end{cases}
\end{aligned}
$$

Similarly, $U_{3}$ and $V_{3}$ satisfy the conditions in Lemma 1.1. Then there exists $x \in \Omega_{3}$ such that $\left(U_{3}+V_{3}\right) x=x$. For $t \in\left[T_{1}, \infty\right)_{\mathbb{T}}$, we obtain

$$
\begin{aligned}
x(t)= & \frac{3\left(1+p_{1} \eta_{3}\right)}{2} H_{3}(t)-p(t) x(g(t)) \\
& +\int_{t}^{\infty} \int_{u_{3}}^{\infty} \int_{u_{2}}^{\infty} \int_{T_{1}}^{u_{1}} \frac{f\left(u_{0}, x\left(h\left(u_{0}\right)\right)\right)}{r_{1}\left(u_{1}\right) r_{2}\left(u_{2}\right) r_{3}\left(u_{3}\right)} \Delta u_{0} \Delta u_{1} \Delta u_{2} \Delta u_{3} .
\end{aligned}
$$

Since

$$
\begin{aligned}
& \int_{t}^{\infty} \int_{u_{3}}^{\infty} \int_{u_{2}}^{\infty} \int_{T_{1}}^{u_{1}} \frac{f\left(u_{0}, x\left(h\left(u_{0}\right)\right)\right)}{r_{1}\left(u_{1}\right) r_{2}\left(u_{2}\right) r_{3}\left(u_{3}\right)} \Delta u_{0} \Delta u_{1} \Delta u_{2} \Delta u_{3} \\
& \quad<H_{3}(t) \int_{T_{1}}^{\infty} f\left(u_{0}, x\left(h\left(u_{0}\right)\right)\right) \Delta u_{0} \leq H_{3}(t) \int_{T_{1}}^{\infty} f\left(u_{0}, 2 H_{3}\left(h\left(u_{0}\right)\right)\right) \Delta u_{0}
\end{aligned}
$$

for $t \in\left[T_{1}, \infty\right)_{\mathbb{T}}$ and

$$
\lim _{t \rightarrow \infty} H_{3}(t) \int_{T_{1}}^{\infty} f\left(u_{0}, 2 H_{3}\left(h\left(u_{0}\right)\right)\right) \Delta u_{0}=0
$$

by virtue of (12), similarly, we can conclude (8) and

$$
R_{0}(t, x(t))>0, \quad R_{1}(t, x(t))<0, \quad R_{2}(t, x(t))>0, \quad R_{3}(t, x(t))<0
$$

for $t \in\left[T_{1}, \infty\right)_{\mathbb{T}}$. This completes the proof.

Secondly, we obtain Theorems 2.5-2.8 based on the assumption that the function $f(t, x)$ satisfies the Lipschitz condition on an interval.

Theorem 2.5 Assume that $H_{1}\left(t_{0}\right)<\infty$. If there exist a constant $L>0$ and two functions $q \in \mathrm{C}_{\mathrm{rd}}\left(\left[t_{0}, \infty\right)_{\mathbb{T}},(0, \infty)\right)$ and $f_{0} \in \mathrm{C}\left(\left[0,2 H_{1}\left(t_{0}\right)\right], \mathbb{R}\right)$ such that

$$
\begin{aligned}
& x f(t, x) \leq x q(t) f_{0}(x), \quad t \in\left[t_{0}, \infty\right)_{\mathbb{T}}, \\
& \left|f\left(t, x_{1}\right)-f\left(t, x_{2}\right)\right| \leq L \cdot q(t)\left|x_{1}-x_{2}\right|, \quad x_{1}, x_{2} \in\left[0,2 H_{1}\left(t_{0}\right)\right],
\end{aligned}
$$

and

$$
\int_{t_{0}}^{\infty} \int_{t_{0}}^{u_{2}} \int_{t_{0}}^{u_{1}} \frac{q\left(u_{0}\right)}{r_{1}\left(u_{1}\right) r_{2}\left(u_{2}\right)} \Delta u_{0} \Delta u_{1} \Delta u_{2}<\infty
$$


then there exists $T_{1} \in\left[t_{0}, \infty\right)_{\mathbb{T}}$ such that (1) has two eventually positive solutions $x_{1}$ and $x_{2}$ tending to zero, which satisfy that $R_{0}\left(t, x_{i}(t)\right)>0, R_{1}\left(t, x_{i}(t)\right)<0, i=1,2, R_{2}\left(t, x_{1}(t)\right)<0$, $R_{3}\left(t, x_{1}(t)\right)<0, R_{2}\left(t, x_{2}(t)\right)>0$, and $R_{3}\left(t, x_{2}(t)\right)>0$ for $t \in\left[T_{1}, \infty\right)_{\mathbb{T}}$.

Proof Take $p_{1}$ satisfying $p_{0}<p_{1}<\left(1+4 p_{0}\right) / 5<1$. There also exists $T_{0} \in\left[t_{0}, \infty\right)_{\mathbb{T}}$ such that (6) holds and

$$
\int_{T_{0}}^{\infty} \int_{T_{0}}^{u_{2}} \int_{T_{0}}^{u_{1}} \frac{q\left(u_{0}\right)}{r_{1}\left(u_{1}\right) r_{2}\left(u_{2}\right)} \Delta u_{0} \Delta u_{1} \Delta u_{2} \leq \min \left\{\frac{1-p_{1} \eta_{1}}{4 K}, 1\right\}
$$

where $K=\max \left\{\left|f_{0}(x)\right|: x \in\left[0,2 H_{1}\left(t_{0}\right)\right]\right\}>0$. Then define the same $T_{1}, \mathrm{BC}\left[T_{0}, \infty\right)_{\mathbb{T}}, \Omega_{1}, U_{1}$, and $V_{1}$ as in Theorem 2.1. Proceeding as in the proof of Theorem 2.1, there exists $x_{1} \in \Omega_{1}$ such that $\left(U_{1}+V_{1}\right) x_{1}=x_{1}$, and we arrive at (7). Since

$$
\begin{gathered}
\int_{t}^{\infty} \int_{T_{1}}^{u_{3}} \int_{T_{1}}^{u_{2}} \int_{T_{1}}^{u_{1}} \frac{f\left(u_{0}, x_{1}\left(h\left(u_{0}\right)\right)\right)}{r_{1}\left(u_{1}\right) r_{2}\left(u_{2}\right) r_{3}\left(u_{3}\right)} \Delta u_{0} \Delta u_{1} \Delta u_{2} \Delta u_{3} \\
<H_{1}(t) \int_{T_{1}}^{\infty} \int_{T_{1}}^{u_{2}} \int_{T_{1}}^{u_{1}} \frac{q\left(u_{0}\right) f_{0}\left(x_{1}\left(h\left(u_{0}\right)\right)\right)}{r_{1}\left(u_{1}\right) r_{2}\left(u_{2}\right)} \Delta u_{0} \Delta u_{1} \Delta u_{2} \\
\leq K \cdot H_{1}(t) \int_{T_{1}}^{\infty} \int_{T_{1}}^{u_{2}} \int_{T_{1}}^{u_{1}} \frac{q\left(u_{0}\right)}{r_{1}\left(u_{1}\right) r_{2}\left(u_{2}\right)} \Delta u_{0} \Delta u_{1} \Delta u_{2}
\end{gathered}
$$

for $t \in\left[T_{1}, \infty\right)_{\mathbb{T}}$ and

$$
\lim _{t \rightarrow \infty} K \cdot H_{1}(t) \int_{T_{1}}^{\infty} \int_{T_{1}}^{u_{2}} \int_{T_{1}}^{u_{1}} \frac{q\left(u_{0}\right)}{r_{1}\left(u_{1}\right) r_{2}\left(u_{2}\right)} \Delta u_{0} \Delta u_{1} \Delta u_{2}=0
$$

in view of (15), by Lemma 1.2, we obtain (8) and

$$
R_{0}\left(t, x_{1}(t)\right)>0, \quad R_{1}\left(t, x_{1}(t)\right)<0, \quad R_{2}\left(t, x_{1}(t)\right)<0, \quad R_{3}\left(t, x_{1}(t)\right)<0
$$

for $t \in\left[T_{1}, \infty\right)_{\mathbb{T}}$. Similarly, we deduce the remaining conclusions as in Theorem 2.1. This completes the proof.

In views of Theorems $2.2-2.5$, we can also obtain Theorems $2.6-2.8$ respectively when $f(t, x)$ satisfies the Lipschitz condition on an interval. The proofs are similar to those of Theorems 2.2-2.4 and thus are omitted.

Theorem 2.6 Assume that $H_{1}\left(t_{0}\right)<\infty$. If there exist a constant $L>0$ and two functions $q \in \mathrm{C}_{\mathrm{rd}}\left(\left[t_{0}, \infty\right)_{\mathbb{T}},(0, \infty)\right)$ and $f_{0} \in \mathrm{C}\left(\left[0,2 H_{1}\left(t_{0}\right)\right], \mathbb{R}\right)$ satisfying $(13)$, (14), and

$$
\int_{t_{0}}^{\infty} \int_{u_{2}}^{\infty} \int_{t_{0}}^{u_{1}} \frac{q\left(u_{0}\right)}{r_{1}\left(u_{1}\right) r_{2}\left(u_{2}\right)} \Delta u_{0} \Delta u_{1} \Delta u_{2}<\infty
$$

then there exists $T_{1} \in\left[t_{0}, \infty\right)_{\mathbb{T}}$ such that (1) has two eventually positive solutions $x_{1}$ and $x_{2}$ tending to zero, which satisfy that $R_{0}\left(t, x_{i}(t)\right)>0, R_{1}\left(t, x_{i}(t)\right)<0, i=1,2, R_{2}\left(t, x_{1}(t)\right)<0$, $R_{3}\left(t, x_{1}(t)\right)>0, R_{2}\left(t, x_{2}(t)\right)>0$, and $R_{3}\left(t, x_{2}(t)\right)<0$ for $t \in\left[T_{1}, \infty\right)_{\mathbb{T}}$. 
Theorem 2.7 Assume that $H_{2}\left(t_{0}\right)<\infty$. If there exist a constant $L>0$ and two functions $q \in \mathrm{C}_{\mathrm{rd}}\left(\left[t_{0}, \infty\right)_{\mathbb{T}},(0, \infty)\right)$ and $f_{0} \in \mathrm{C}\left(\left[0,2 H_{2}\left(t_{0}\right)\right], \mathbb{R}\right)$ satisfying $(13)$,

$$
\left|f\left(t, x_{1}\right)-f\left(t, x_{2}\right)\right| \leq L \cdot q(t)\left|x_{1}-x_{2}\right|, \quad x_{1}, x_{2} \in\left[0,2 H_{2}\left(t_{0}\right)\right],
$$

and

$$
\int_{t_{0}}^{\infty} \int_{t_{0}}^{u_{1}} \frac{q\left(u_{0}\right)}{r_{1}\left(u_{1}\right)} \Delta u_{0} \Delta u_{1}<\infty
$$

then there exists $T_{1} \in\left[t_{0}, \infty\right)_{\mathbb{T}}$ such that (1) has two eventually positive solutions $x_{1}$ and $x_{2}$ tending to zero, which satisfy that $R_{0}\left(t, x_{i}(t)\right)>0, R_{1}\left(t, x_{i}(t)\right)<0, R_{2}\left(t, x_{i}(t)\right)>0, i=1,2$, $R_{3}\left(t, x_{1}(t)\right)>0$, and $R_{3}\left(t, x_{2}(t)\right)<0$ for $t \in\left[T_{1}, \infty\right)_{\mathbb{T}}$.

Theorem 2.8 Assume that $H_{3}\left(t_{0}\right)<\infty$. If there exist a constant $L>0$ and two functions $q \in \mathrm{C}_{\mathrm{rd}}\left(\left[t_{0}, \infty\right)_{\mathbb{T}},(0, \infty)\right)$ and $f_{0} \in \mathrm{C}\left(\left[0,2 H_{3}\left(t_{0}\right)\right], \mathbb{R}\right)$ satisfying $(13)$,

$$
\left|f\left(t, x_{1}\right)-f\left(t, x_{2}\right)\right| \leq L \cdot q(t)\left|x_{1}-x_{2}\right|, \quad x_{1}, x_{2} \in\left[0,2 H_{3}\left(t_{0}\right)\right],
$$

and

$$
\int_{t_{0}}^{\infty} q\left(u_{0}\right) \Delta u_{0}<\infty
$$

then there exists $T_{1} \in\left[t_{0}, \infty\right)_{\mathbb{T}}$ such that (1) has an eventually positive solution $x$ tending to zero, which satisfies that $R_{0}(t, x(t))>0, R_{1}(t, x(t))<0, R_{2}(t, x(t))>0$, and $R_{3}(t, x(t))<0$ for $t \in\left[T_{1}, \infty\right)_{\mathbb{T}}$.

In addition, we also have the following conclusion.

Theorem 2.9 Assume that one of the following conditions

$$
\begin{aligned}
& \int_{t_{0}}^{\infty} \frac{\Delta u_{3}}{r_{3}\left(u_{3}\right)}=\infty, \\
& \int_{t_{0}}^{\infty} \int_{u_{3}}^{\infty} \frac{\Delta u_{2} \Delta u_{3}}{r_{2}\left(u_{2}\right) r_{3}\left(u_{3}\right)}=\infty,
\end{aligned}
$$

and

$$
\int_{t_{0}}^{\infty} \int_{u_{3}}^{\infty} \int_{u_{2}}^{\infty} \frac{\Delta u_{1} \Delta u_{2} \Delta u_{3}}{r_{1}\left(u_{1}\right) r_{2}\left(u_{2}\right) r_{3}\left(u_{3}\right)}=\infty
$$

holds, then (1) has no eventually positive solution $x$, for which $R_{1}, R_{2}$, and $R_{3}$ are all eventually negative.

Proof Suppose that $x$ is an eventually positive solution of $(1)$ and there exists $T_{0} \in\left[t_{0}, \infty\right)_{\mathbb{T}}$ such that, for $t \in\left[T_{0}, \infty\right)_{\mathbb{T}}$, we have

$$
x(t)>0, \quad R_{1}(t, x(t))<0, \quad R_{2}(t, x(t))<0, \quad R_{3}(t, x(t))<0 .
$$


There also exists $T_{1} \in\left(T_{0}, \infty\right)_{\mathbb{T}}$ such that $g(t) \geq T_{0}$ and $h(t) \geq T_{0}$ for $t \in\left[T_{1}, \infty\right)_{\mathbb{T}}$. Substituting $u_{0}$ for $t$ in (1) and integrating (1) with respect to $u_{0}$ from $T_{1}$ to $u_{1}$, where $u_{1} \in\left[\sigma\left(T_{1}\right), \infty\right)_{\mathbb{T}}$, we have

$$
R_{3}\left(u_{1}, x\left(u_{1}\right)\right)-R_{3}\left(T_{1}, x\left(T_{1}\right)\right)=-\int_{T_{1}}^{u_{1}} f\left(u_{0}, x\left(h\left(u_{0}\right)\right)\right) \Delta u_{0}<0,
$$

which implies that

$$
R_{2}^{\Delta}\left(u_{1}, x\left(u_{1}\right)\right)<\frac{R_{3}\left(T_{1}, x\left(T_{1}\right)\right)}{r_{1}\left(u_{1}\right)} .
$$

Integrating (19) with respect to $u_{1}$ from $T_{1}$ to $u_{2}$, where $u_{2} \in\left[\sigma\left(T_{1}\right), \infty\right)_{\mathbb{T}}$, we have

$$
R_{2}\left(u_{2}, x\left(u_{2}\right)\right)<R_{2}\left(T_{1}, x\left(T_{1}\right)\right)+R_{3}\left(T_{1}, x\left(T_{1}\right)\right) \int_{T_{1}}^{u_{2}} \frac{\Delta u_{1}}{r_{1}\left(u_{1}\right)} .
$$

By analogy, we obtain

$$
\begin{aligned}
R_{0}(t, x(t))< & R_{0}\left(T_{1}, x\left(T_{1}\right)\right)+R_{1}\left(T_{1}, x\left(T_{1}\right)\right) \int_{T_{1}}^{t} \frac{\Delta u_{3}}{r_{3}\left(u_{3}\right)} \\
& +R_{2}\left(T_{1}, x\left(T_{1}\right)\right) \int_{T_{1}}^{t} \int_{T_{1}}^{u_{3}} \frac{\Delta u_{2} \Delta u_{3}}{r_{2}\left(u_{2}\right) r_{3}\left(u_{3}\right)} \\
& +R_{3}\left(T_{1}, x\left(T_{1}\right)\right) \int_{T_{1}}^{t} \int_{T_{1}}^{u_{3}} \int_{T_{1}}^{u_{2}} \frac{\Delta u_{1} \Delta u_{2} \Delta u_{3}}{r_{1}\left(u_{1}\right) r_{2}\left(u_{2}\right) r_{3}\left(u_{3}\right)} .
\end{aligned}
$$

If one of (16)-(18) holds, then we derive $R_{0}(t, x(t)) \rightarrow-\infty$ as $t \rightarrow \infty$. However, we have $R_{0}(t, x(t))=x(t)+p(t) x(g(t))>0$ for $t \in\left[T_{1}, \infty\right)_{\mathbb{T}}$. It causes a contradiction. This completes the proof.

\section{Examples}

In this section, two interesting examples are provided to illustrate the conclusions.

Example 3.1 Let $\mathbb{T}=\bigcup_{n=1}^{\infty}[2 n-1,2 n]$. For $t \in[3, \infty)_{\mathbb{T}}$, consider

$$
\left(t^{\alpha}\left(t^{\beta}\left(t^{2}\left(x(t)+p(t) x\left(t-\frac{\cos \pi t}{\pi}\right)\right)^{\Delta}\right)^{\Delta}\right)^{\Delta}\right)^{\Delta}+t \cdot x(t-2)=0,
$$

where $p$ satisfies (C2). Here, we have $r_{1}(t)=t^{\alpha}, r_{2}(t)=t^{\beta}, r_{3}(t)=t^{2}, g(t)=t-\cos (\pi t) / \pi$, $h(t)=t-2, f(t, x)=t \cdot x$, and $t_{0}=3$. Moreover, we obtain

$$
\int_{t_{0}}^{\infty} \frac{\Delta u_{3}}{r_{3}\left(u_{3}\right)}=\int_{3}^{\infty} \frac{\Delta u_{3}}{u_{3}^{2}}<\infty, \quad H_{1}(t)=\int_{t}^{\infty} \frac{\Delta u_{3}}{u_{3}^{2}}<\frac{1}{2}, \quad t \in[3, \infty)_{\mathbb{T}},
$$

and $\eta_{1}=\lim _{t \rightarrow \infty} H_{1}(g(t)) / H_{1}(t)=1$. Hence, it fulfills conditions $(\mathrm{C} 1)-(\mathrm{C} 5)$. Since $f(t, x)$ is nondecreasing with respect to $x$, when $\alpha>3$ and $\beta>1$, or $\alpha \leq 3$ and $\beta>4-\alpha$, we have

$$
\int_{t_{0}}^{\infty} \int_{t_{0}}^{u_{2}} \int_{t_{0}}^{u_{1}} \frac{f\left(u_{0}, 2 H_{1}\left(h\left(u_{0}\right)\right)\right)}{r_{1}\left(u_{1}\right) r_{2}\left(u_{2}\right)} \Delta u_{0} \Delta u_{1} \Delta u_{2}
$$




$$
\leq \int_{3}^{\infty} \int_{3}^{u_{2}} \int_{3}^{u_{1}} \frac{u_{0}}{u_{1}^{\alpha} u_{2}^{\beta}} \Delta u_{0} \Delta u_{1} \Delta u_{2}<\frac{1}{2} \int_{3}^{\infty} \int_{3}^{u_{2}} \frac{\Delta u_{1} \Delta u_{2}}{u_{1}^{\alpha-2} u_{2}^{\beta}}<\infty
$$

which means that (20) has two eventually positive solutions $x_{1}$ and $x_{2}$ tending to zero in terms of Theorem 2.1. Moreover, there exists $T_{1} \in\left[t_{0}, \infty\right)_{\mathbb{T}}$ such that $R_{0}\left(t, x_{i}(t)\right)>0$, $R_{1}\left(t, x_{i}(t)\right)<0, i=1,2, R_{2}\left(t, x_{1}(t)\right)<0, R_{3}\left(t, x_{1}(t)\right)<0, R_{2}\left(t, x_{2}(t)\right)>0$, and $R_{3}\left(t, x_{2}(t)\right)>0$ for $t \in\left[T_{1}, \infty\right)_{\mathbb{T}}$.

When $\alpha>3$ and $\beta>4-\alpha$, it follows that

$$
\int_{t_{0}}^{\infty} \int_{u_{2}}^{\infty} \int_{t_{0}}^{u_{1}} \frac{f\left(u_{0}, 2 H_{1}\left(h\left(u_{0}\right)\right)\right)}{r_{1}\left(u_{1}\right) r_{2}\left(u_{2}\right)} \Delta u_{0} \Delta u_{1} \Delta u_{2}<\frac{1}{2} \int_{3}^{\infty} \int_{u_{2}}^{\infty} \frac{\Delta u_{1} \Delta u_{2}}{u_{1}^{\alpha-2} u_{2}^{\beta}}<\infty .
$$

Hence, we deduce that (20) has two eventually positive solutions satisfying the conclusions of Theorem 2.2.

When $\alpha>3$ and $\beta>1$, we obtain $H_{2}(3)<\infty$. Then there exists a constant $M>0$ such that

$$
H_{2}(t)=\int_{t}^{\infty} \int_{u_{3}}^{\infty} \frac{\Delta u_{2} \Delta u_{3}}{u_{2}^{\beta} u_{3}^{2}} \leq M
$$

from which it follows that

$$
\begin{aligned}
& \int_{t_{0}}^{\infty} \int_{t_{0}}^{u_{1}} \frac{f\left(u_{0}, 2 H_{2}\left(h\left(u_{0}\right)\right)\right)}{r_{1}\left(u_{1}\right)} \Delta u_{0} \Delta u_{1} \\
& \quad \leq 2 M \int_{3}^{\infty} \int_{3}^{u_{1}} \frac{u_{0}}{u_{1}^{\alpha}} \Delta u_{0} \Delta u_{1}<4 M \int_{3}^{\infty} \frac{\Delta u_{1}}{u_{1}^{\alpha-2}}<\infty .
\end{aligned}
$$

Note that $\eta_{2}=1$. Therefore, (20) has two eventually positive solutions fulfilling the results of Theorem 2.3.

When $\alpha>1$ and $\beta>3-\alpha$, we obtain $H_{3}(3)<\infty$,

$$
H_{3}(t)=\int_{t}^{\infty} \int_{u_{3}}^{\infty} \int_{u_{2}}^{\infty} \frac{\Delta u_{1} \Delta u_{2} \Delta u_{3}}{u_{1}^{\alpha} u_{2}^{\beta} u_{3}^{2}}=O\left(t^{1-\alpha-\beta}\right), \quad \eta_{3}=1
$$

and

$$
\int_{t_{0}}^{\infty} f\left(u_{0}, 2 H_{3}\left(h\left(u_{0}\right)\right)\right) \Delta u_{0}=\int_{3}^{\infty} O\left(\frac{1}{u_{0}^{\alpha+\beta-2}}\right) \Delta u_{0}<\infty
$$

where the conclusions of Theorem 2.4 are satisfied.

On the other hand, consider conditions (16)-(18). Obviously, (16) does not hold here. Then (17) is satisfied when $\alpha \in \mathbb{R}$ and $\beta \leq 1$, and (18) holds when $\alpha \leq 1$ and $\beta \in \mathbb{R}$, or $\alpha>1$ and $\beta \leq 2-\alpha$. By virtue of Theorem 2.9, if these conditions of $\alpha$ and $\beta$ are satisfied, then we can conclude that (20) has no eventually positive solution $x$, for which $R_{1}, R_{2}$, and $R_{3}$ are all eventually negative.

Example 3.2 Let $\mathbb{T}=[1, \infty)_{\mathbb{R}}$. For $t \in \mathbb{T}$, consider

$$
\left(t^{\alpha}\left(t^{\beta}\left(t^{3}(x(t)+p(t) x(t+1))^{\prime}\right)^{\prime}\right)^{\prime}\right)^{\prime}+\frac{x^{3}(t)}{t}=0
$$


where $p$ satisfies (C2). Here, we have $r_{1}(t)=t^{\alpha}, r_{2}(t)=t^{\beta}, r_{3}(t)=t^{3}, g(t)=t+1, h(t)=t$, $f(t, x)=x^{3} / t$, and $t_{0}=1$. Then, we take $q(t)=1 / t$ and $f_{0}(x)=x^{3}$.

Firstly, for $\alpha \geq 1$ and $\beta>1$, we have

$$
\begin{aligned}
H_{1}\left(t_{0}\right)=\int_{t_{0}}^{\infty} \frac{d u_{3}}{r_{3}\left(u_{3}\right)} & =\int_{1}^{\infty} \frac{d u_{3}}{u_{3}^{3}}=\frac{1}{2}, \quad \eta_{1}=1, \\
\left|f\left(t, x_{1}\right)-f\left(t, x_{2}\right)\right| & =\frac{1}{t} \cdot\left|x_{1}-x_{2}\right| \cdot\left|x_{1}^{2}+x_{1} x_{2}+x_{2}^{2}\right| \\
& \leq 3 \cdot \frac{1}{t} \cdot\left|x_{1}-x_{2}\right|, \quad x_{1}, x_{2} \in[0,1],
\end{aligned}
$$

and

$$
\int_{t_{0}}^{\infty} \int_{t_{0}}^{u_{2}} \int_{t_{0}}^{u_{1}} \frac{q\left(u_{0}\right)}{r_{1}\left(u_{1}\right) r_{2}\left(u_{2}\right)} d u_{0} d u_{1} d u_{2}=\int_{1}^{\infty} \int_{1}^{u_{2}} \int_{1}^{u_{1}} \frac{d u_{0} d u_{1} d u_{2}}{u_{0} u_{1}^{\alpha} u_{2}^{\beta}}<\infty
$$

from which we get the conclusion of Theorem 2.5. On the other hand, for $\alpha>1$ and $\beta \geq 1$, we derive

$$
\int_{t_{0}}^{\infty} \int_{u_{2}}^{\infty} \int_{t_{0}}^{u_{1}} \frac{q\left(u_{0}\right)}{r_{1}\left(u_{1}\right) r_{2}\left(u_{2}\right)} d u_{0} d u_{1} d u_{2}=\int_{1}^{\infty} \int_{u_{2}}^{\infty} \int_{1}^{u_{1}} \frac{d u_{0} d u_{1} d u_{2}}{u_{0} u_{1}^{\alpha} u_{2}^{\beta}}<\infty
$$

Hence, the result of Theorem 2.6 is obtained.

Secondly, for $\alpha>1$ and $\beta>1$, there exists a constant $M>0$ such that

$$
H_{2}\left(t_{0}\right)=\int_{t_{0}}^{\infty} \int_{u_{3}}^{\infty} \frac{d u_{2} d u_{3}}{r_{2}\left(u_{2}\right) r_{3}\left(u_{3}\right)}=\int_{1}^{\infty} \int_{u_{3}}^{\infty} \frac{d u_{2} d u_{3}}{u_{2}^{\beta} u_{3}^{3}} \leq M, \quad \eta_{2}=1 .
$$

Moreover, it follows that

$$
\left|f\left(t, x_{1}\right)-f\left(t, x_{2}\right)\right| \leq 12 M^{2} \cdot \frac{1}{t} \cdot\left|x_{1}-x_{2}\right|, \quad x_{1}, x_{2} \in[0,2 M]
$$

and

$$
\int_{t_{0}}^{\infty} \int_{t_{0}}^{u_{1}} \frac{q\left(u_{0}\right)}{r_{1}\left(u_{1}\right)} d u_{0} d u_{1}=\int_{1}^{\infty} \int_{1}^{u_{1}} \frac{d u_{0} d u_{1}}{u_{0} u_{1}^{\alpha}}<\infty .
$$

Then we obtain the conclusion of Theorem 2.7.

Finally, we find that

$$
\int_{t_{0}}^{\infty} q\left(u_{0}\right) d u_{0}=\int_{1}^{\infty} \frac{d u_{0}}{u_{0}}=\infty
$$

so the result of Theorem 2.8 seems not to be deduced. However, for $\alpha>1$ and $\beta>1$, in view of Theorem 2.4, we have $H_{3}(1)<\infty$,

$$
H_{3}(t)=\int_{t}^{\infty} \int_{u_{3}}^{\infty} \int_{u_{2}}^{\infty} \frac{d u_{1} d u_{2} d u_{3}}{u_{1}^{\alpha} u_{2}^{\beta} u_{3}^{3}}=O\left(t^{-\alpha-\beta}\right), \quad \eta_{3}=1,
$$


and

$$
\int_{t_{0}}^{\infty} f\left(u_{0}, 2 H_{3}\left(h\left(u_{0}\right)\right)\right) d u_{0}=\int_{3}^{\infty} \frac{d u_{0}}{O\left(u_{0}^{3 \alpha+3 \beta+1}\right)}<\infty
$$

Therefore, we still derive the result of Theorem 2.4 (or Theorem 2.8).

\section{Conclusion}

In this paper, we successfully obtain some new results for the existence of nonoscillatory solutions tending to zero of a class of fourth-order nonlinear neutral dynamic equations on time scales. Moreover, compared with the existing references, the assumptions of functions $f$ and $g$ are more relaxed. According to this technique, we can continue to study the existence of nonoscillatory solutions tending to zero of similar forms of higher-order nonlinear neutral dynamic equations on time scales.

\section{Acknowledgements}

The author thanks the anonymous referees for their valuable suggestions.

Funding

This project was supported by the National Natural Science Foundation of China (11671406 and 12071491) and the Innovation Enhancing College Project of Department of Education of Guangdong Province (2020KTSCX367).

\section{Availability of data and materials}

Not applicable.

\section{Competing interests}

The author declares that he has no competing interests.

Authors' contributions

All authors read and approved the final manuscript.

\section{Publisher's Note}

Springer Nature remains neutral with regard to jurisdictional claims in published maps and institutional affiliations.

Received: 18 November 2020 Accepted: 28 July 2021 Published online: 06 August 2021

\section{References}

1. Hilger, S.: Ein Maßkettenkalkül mit Anwendung auf Zentrumsmannigfaltigkeiten. Ph.D. thesis, Universität Würzburg (1988)

2. Hilger, S.: Analysis on measure chains - a unified approach to continuous and discrete calculus. Results Math. 18, 18-56 (1990)

3. Agarwal, R.P. Bohner, M.: Basic calculus on time scales and some of its applications. Results Math. 35, 3-22 (1999)

4. Agarwal, R.P., Bohner, M., O'Regan, D., Peterson, A.: Dynamic equations on time scales: a survey. J. Comput. Appl. Math. 141, 1-26 (2002)

5. Bohner, M., Peterson, A.: Dynamic Equations on Time Scales: An Introduction with Applications. Birkhäuser, Boston (2001)

6. Bohner, M., Peterson, A.: Advances in Dynamic Equations on Time Scales. Birkhäuser, Boston (2003)

7. Deng, X.-H., Wang, Q.-R.: Nonoscillatory solutions to second-order neutral functional dynamic equations on time scales. Commun. Appl. Anal. 18, 261-280 (2014)

8. Gao, J., Wang, Q.R.: Existence of nonoscillatory solutions to second-order nonlinear neutral dynamic equations on time scales. Rocky Mt. J. Math. 43, 1521-1535 (2013)

9. Karpuz, B.: Asymptotic behaviour of bounded solutions of a class of higher-order neutral dynamic equations. Appl. Math. Comput. 215, 2174-2183 (2009)

10. Karpuz, B.: Necessary and sufficient conditions on the asymptotic behaviour of second-order neutral delay dynamic equations with positive and negative coefficients. Math. Methods Appl. Sci. 37, 1219-1231 (2014)

11. Karpuz, B., Öcalan, Ö.: Necessary and sufficient conditions on asymptotic behaviour of solutions of forced neutral delay dynamic equations. Nonlinear Anal. 71, 3063-3071 (2009)

12. Karpuz, B., Öcalan, Ö., Rath, R.: Necessary and sufficient conditions for the oscillatory and asymptotic behaviour of solutions to neutral delay dynamic equations. Electron. J. Differ. Equ. 2009, 64 (2009)

13. Li, T.X., Han, Z.L., Sun, S.R., Yang, D.W.: Existence of nonoscillatory solutions to second-order neutral delay dynamic equations on time scales. Adv. Differ. Equ. 2009, 562329 (2009)

14. Li, T.X. Zhang, C.H. Thandapani, E.: Asymptotic behavior of fourth-order neutral dynamic equations with noncanonical operators. Taiwan. J. Math. 18, 1003-1019 (2014) 
15. Qiu, Y.-C.: Nonoscillatory solutions to third-order neutral dynamic equations on time scales. Adv. Differ. Equ. 2014, 309 (2014)

16. Qiu, Y.-C.: Nonoscillatory solutions to fourth-order neutral dynamic equations on time scales. Adv. Differ. Equ. 2019, $512(2019)$

17. Qiu, Y.-C., Chiu, K.-S., Jadlovská, I., Li, T.X.: Existence of nonoscillatory solutions to nonlinear higher-order neutral dynamic equations. Adv. Differ. Equ. 2020, 475 (2020)

18. Qiu, Y.-C., Jadlovská, I., Lassoued, D., Li, T.X.: Nonoscillatory solutions to higher-order nonlinear neutral dynamic equations. Symmetry 11, 302 (2019)

19. Qiu, Y.-C., Wang, H.X., Jiang, C.M., Li, T.X.: Existence of nonoscillatory solutions to third-order neutral functional dynamic equations on time scales. J. Nonlinear Sci. Appl. 11, 274-287 (2018)

20. Qiu, Y.-C., Wang, Q.-R.: Existence of nonoscillatory solutions to higher-order nonlinear neutral dynamic equations on time scales. Bull. Malays. Math. Sci. Soc. 41, 1935-1952 (2018)

21. Qiu, Y.-C., Zada, A., Tang, S.H., Li, T.X.: Existence of nonoscillatory solutions to nonlinear third-order neutral dynamic equations on time scales. J. Nonlinear Sci. Appl. 10, 4352-4363 (2017)

22. Zhu, Z.-Q., Wang, Q.-R.: Existence of nonoscillatory solutions to neutral dynamic equations on time scales. J. Math. Anal. Appl. 335, 751-762 (2007)

23. Mojsej, I., Tartal'ová, A.: On nonoscillatory solutions tending to zero of third-order nonlinear differential equations. Tatra Mt. Math. Publ. 48, 135-143 (2011)

24. Qiu, Y.-C.: On nonoscillatory solutions tending to zero of third-order nonlinear dynamic equations on time scales. Adv. Differ. Equ. 2016, 10 (2016)

25. Qiu, Y.-C., Jadlovská, I., Chiu, K.-S., Li, T.X.: Existence of nonoscillatory solutions tending to zero of third-order neutral dynamic equations on time scales. Adv. Differ. Equ. 2020, 231 (2020)

26. Chen, Y.S.: Existence of nonoscillatory solutions of $n$th order neutral delay differential equations. Funcialaj Ekvacioj 35 557-570 (1992)

\section{Submit your manuscript to a SpringerOpen ${ }^{\circ}$ journal and benefit from:}

- Convenient online submission

- Rigorous peer review

- Open access: articles freely available online

- High visibility within the field

- Retaining the copyright to your article

Submit your next manuscript at $\boldsymbol{\nabla}$ springeropen.com 